\title{
Development of a Type of Non-Contact Driver in an Underwater Rope Drum
}

\author{
Bo ZHANG ${ }^{1}$, Zhuo WANG ${ }^{1, a}$ and Tao WANG $^{2}$ \\ ${ }^{1}$ Harbin Engineering University, College of Mechanical and Electrical Engineering, 150001Harbin, \\ China \\ ${ }^{2}$ Hebei University of Technology, School of MechanicalEngineering, 300401Tianjin, China \\ ajlwzh@163.com
}

Keywords: Dynamic seals, Experiments in pulling rope, Non-contact driver, Magnetic materials, Rope drum.

\begin{abstract}
Research on a type of non-contact driver made of magnetic materials in a rope drum was carried out to solve the problem of leakage in the deep dynamic seals of motor output shafts. An isolation sleeve was added between the inner and outer magnetic rotor, which changed the sealing type from a dynamic seal located on the motor output shaft to a static seal on the front end; this allows the sealing depth to go down more than $10000 \mathrm{~m}$. This study designed the driver components, analyzed the principle of magnetic coupling, and used the method of finite elements to analyse the intensity of the sleeve. Through the rope drum sealing pressure test and the pulling rope experiments at sea, its sealing pressure can reach up to $110 \mathrm{MPa}$. Research verified the effectiveness of the sealing, rotational stability, tensioning reliability, and engineering feasibility.
\end{abstract}

\section{Introduction}

The rope drum refers to the crane and hoist drum, the winch used for cable winding, the rotating mechanism for coiling soft rope, the hose, etc., which can be used to wind rope within the scope of water depths of 1500 10000 meters. It is the key technology for sealing the motor output shaft about the rotation of its shaft in deepwater [1].There is four main structure kinds of underwater dynamic seals: the o-ring seal, combination seal, mechanical seal, and magnetic liquid seal. The sealing pressure on the general rubber o-ring cannot be more than $1.5 \mathrm{MPa}$, and its rotating velocity is less than $3.5 \mathrm{~m} / \mathrm{s}$ [2]. The sealing pressure on the combined seal is less than $70 \mathrm{MPa}$, and its rotating velocity is less than $6 \mathrm{~m} / \mathrm{s}$. The single-stage magnetic liquid seal has a maximum capacity for $120 \mathrm{kPa}$ to $180 \mathrm{kPa}$, and the whole sealing pressure is less than $1.5 \mathrm{MPa}$ at a depth of 150 meters [3-5]. The sealing pressure of the mechanical seal reaches up to $45 \mathrm{MPa}$, and its rotation speed can achieve up to $150 \mathrm{~m} / \mathrm{s}$ [6].As water depth increases, the more and bigger pressure will act on the motor output shaft seal. When the depth is more than 4500 meters, an ordinary dynamic seal will leak and cannot achieve the containment requirements. For magnetic materials used to transmit torque, it needs not only high magnetic induction intensity, but also a high coercive force and magnetic energy product. There are kinds of ferrite magnet steel and rare earth magnet steel that meet the torque requirements of magnetic materials. So far, the third generation of rare earth permanent magnet $\mathrm{Nd}-\mathrm{Fe}-\mathrm{B}$ is the superior magnetic energy product. Because Nd-Fe-B magnetic materials have excellent magnetism and a low price, they can obtain satisfactory magnetic torque on the structure of magnetic circuits through cylindrical combination. Their unique advantages are that they have a small volume, light weight, large power, and high efficiency; therefore, they are widely used in corrosion environments[7].This paper will use magnetic materials to research a driver unconnected in a rope drum under water and solve the difficulty of getting a dynamic seal on the motor output shaft[8]. It will also carry out experiments to test the effectiveness of a dynamic seal and whether the driving moment meets the requirements of the pulling rope. 


\section{Method of Experiments on the Pulling Rope}

The action of a rope drum involves pulling tightly or taking back rope of a certain length, so one needs to know how high tension is at this moment. The rope used for this test has a diameter of $3 \mathrm{~mm}$, a length of $30 \mathrm{~m}$, and is made of material $1 \mathrm{CrNi}$ Ti. The test method fixes one end of the rope and uses a spring balance to exert different tensions $F_{\mathrm{T}}$ on the other end of the rope; meanwhile, it uses the metric scale to measure the corresponding sag $f$ on different spans, which are $L=5 \mathrm{~m}, 10 \mathrm{~m}, 20 \mathrm{~m}$, $30 \mathrm{~m}$. The four groups of data are shown in Table 1 .

Table 1. The data of test about tension $F_{T}$ and $\operatorname{sag} f$

\begin{tabular}{|c|c|c|c|c|c|c|c|c|c|c|c|c|c|c|c|c|c|}
\hline $\boldsymbol{L}(\mathbf{m})$ & \multicolumn{4}{|c|}{$\mathbf{5}$} & \multicolumn{4}{|c|}{$\mathbf{1 0}$} & \multicolumn{4}{c|}{$\mathbf{3 0} \mathbf{~}$} \\
\hline $\boldsymbol{F}_{\boldsymbol{T}}(\mathbf{N})$ & 2.4 & 10 & 20 & 29 & 10 & 29 & 59 & 78 & 123 & 157 & 183 & 216 & 67 & 88 & 108 & 216 & 376 \\
\hline $\boldsymbol{f}(\mathbf{c m})$ & 17 & 7 & 3 & 0.1 & 29 & 9 & 2 & 0.1 & 10 & 7 & 4 & 1 & 53 & 43 & 34 & 14 & 0.1 \\
\hline
\end{tabular}

Fig. 1 shows the device for the experiments on the pulling rope. Figure 2 shows the curve of tension and the sag. Fig. 2 shows the curve of tension and the sag.

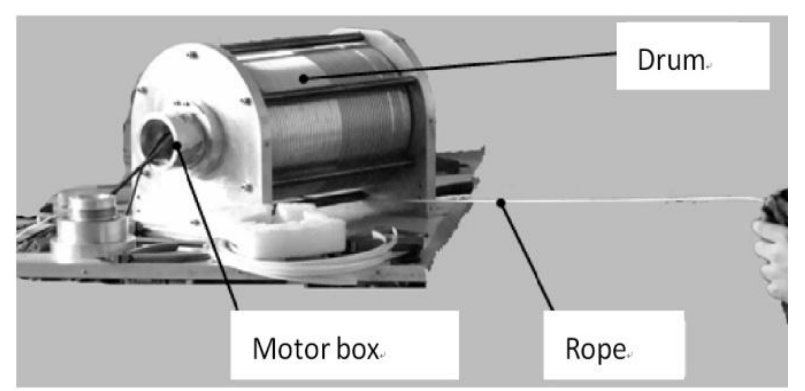

Fig. 1.Experiments on pulling rope

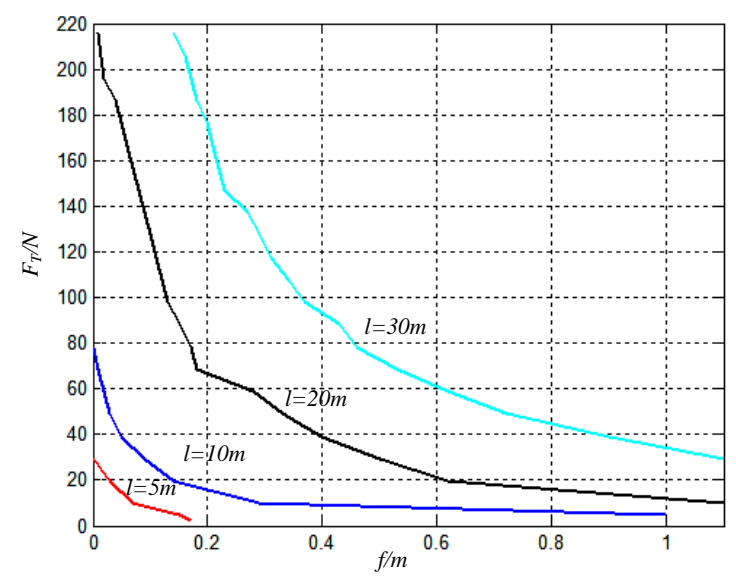

Fig. 2.The curves of tests about rope tension and sag

\section{Results and Design of the Magnetic Driver}

\section{Results of Experiments on Pulling Rope}

The ability to develop the torque of certain magnitude will be formulated .According to the experimental data in Table 1 and Figure 2, when the spans are $L=5,10$ and $20 \mathrm{~m}$ separately, each group has four sets of data, so a quadratic polynomial can fit the curve. The quadratic polynomial form is $y=a_{2} x^{2}+a_{1} x+a_{0}$; it needs more than three groups to solve the parameters $a_{0}, a_{1}$, and $a_{2}$. We can get formulas (1), but when the span is $L=30 \mathrm{~m}$, the data involves more than four groups, and the independent variable has relatively large variable ranges, so the formulas (1)which are of the quadratic polynomial form cannot allow to asses curve fitting quality. Using the fitting method of 
high order polynomials may ensure that the curve fits precisely. Data analysis showed that using four polynomials to fit the curve can reflect the changing rule of the experimental data better. When the spans are $L=5 \mathrm{~m}, 10 \mathrm{~m}, 20 \mathrm{~m}$, and $30 \mathrm{~m}$ separately, the rope tension formula may be written, as [9]

$$
\begin{aligned}
& F_{T}{ }^{\prime}=\left\{\begin{array}{lc}
1833.3 f^{2}-404.2 f+29.6 & (0 \leq f \leq 0.1 m, L=5 m) \\
6522.5 f^{2}-1132.2 f+78.6 & (0 \leq f \leq 0.1 m, L=10 m) \\
-1113.6 f^{2}-884.2 f+223.9 & (0 \leq f \leq 0.1 m, L=20 m)
\end{array}\right. \\
& F_{T}{ }^{\prime}=746.9 f^{4}-2317.4 f^{3}+2694.2 f^{2} \\
& -1469.1 f+376.6(f \geq 0, L=30 m)
\end{aligned}
$$

From formulas (1) and (2), we can get the calculated value of tension size, which can be obtained under the different spans corresponding to line sag $f$ shown in Table 2. Although there have been some errors between measured values and calculated values, but these errors ratio are all withinthelimits $2 \%$. So, we can apply the formulas (1) and (2) to calculate the $3 \mathrm{~mm}$ wire rope tension at other different spans, and provide the data of tension for designing a magnetic driver.

Table 2.The rope tension needed for different sags

\begin{tabular}{|l|c|c|c|c|}
\hline Sag $\boldsymbol{f}(\mathbf{c m})$ & \multicolumn{4}{|c|}{ Tension $\boldsymbol{F}_{\boldsymbol{T}}(\mathbf{N})$} \\
\hline & $L=500$ & $L=1000$ & $L=2000$ & $L=3000$ \\
\hline 0.1 & 30.0 & 80.0 & 230.0 & 380.0 \\
\hline 1 & 25.7 & 67.9 & 214.9 & 361.6 \\
\hline 5 & 13.9 & 38.3 & 176.9 & 309.6 \\
\hline 10 & 7.5 & 30.6 & 124.3 & 254.4 \\
\hline 20 & 3.9 & 15.4 & 61.3 & 173.2 \\
\hline 30 & 2.6 & 10.3 & 40.9 & 121.8 \\
\hline
\end{tabular}

\section{Effect of the Permanent Magnet Materials}

The permanent magnet materials of the driver will be of such nature of high magnetic energy product and difficult to remove magnetization. Table 3 shows the relation between the permanent magnet materials and its torque when $t_{\mathrm{g}}=0.5 \mathrm{~cm}, t_{\mathrm{m}}=0.5 \mathrm{~cm}$ and $\theta=7.5[10]$.

Table 3. The value of permanent magnet materials and torque

\begin{tabular}{|c|c|c|c|c|c|c|}
\hline Magnet materials & $\mathbf{N}_{\mathbf{d}} \mathbf{F}_{\mathbf{e}} \mathbf{B}_{\mathbf{4 0}}$ & $\mathbf{N}_{\mathbf{d}} \mathbf{F}_{\mathbf{e}} \mathbf{B}_{\mathbf{3 7}}$ & $\mathbf{N}_{\mathbf{d}} \mathbf{F}_{\mathbf{e}} \mathbf{B}_{\mathbf{3 2}}$ & $\mathbf{N}_{\mathbf{d}} \mathbf{F}_{\mathrm{e}} \mathbf{B}_{\mathbf{1 0}}$ & $\mathbf{S}_{\mathbf{m}} \mathbf{C}_{\mathbf{0 2 7}}$ & $\mathbf{S}_{\mathbf{m}} \mathbf{C}_{\mathbf{0 2 4}}$ \\
\hline$T(\mathrm{~N} \cdot \mathrm{m})$ & 41.21 & 38.4 & 35.8 & 2.75 & 33.2 & 29.9 \\
\hline
\end{tabular}

This type of driver chooses Ne-Fe-B permanent magnet materials to make the internal and external rotors. Its physical properties are shown in Table 4[10]

Table 4. Properties and physical properties of Nd-Fe-B

\begin{tabular}{|c|c|c|c|}
\hline Residual magnetization $\boldsymbol{B}_{\mathbf{r}}$ & Coercive force $\boldsymbol{H}_{\mathbf{c B}}$ & Intrinsic field $\boldsymbol{H}_{\mathbf{c j}}$ & energy product $(\boldsymbol{B H})_{\max }$ \\
\hline $\mathrm{T}$ & $\mathrm{kA} / \mathrm{m}$ & $\mathrm{kA} / \mathrm{m}$ & $\mathrm{kJ} / \mathrm{m}^{3}$ \\
\hline$\geq 1.17$ & $\geq 844$ & $\geq 1595$ & $247 \sim 263$ \\
\hline Density $d$ & Resistance $\rho$ & Bending strength & Compressive strength \\
\hline $\mathrm{g} / \mathrm{cm}^{3}$ & $\Omega \cdot \mathrm{m}$ & $\mathrm{MPa}$ & $\mathrm{MPa}$ \\
\hline 7.4 & 14400 & 245 & 735 \\
\hline
\end{tabular}

\section{Design of Magnetic Driver Structure}

A magnetic driver is new type of driver that connects the work machine to the prime mover used with the magnetic force of a permanent magnet. It needn't connect the rotator in direct mechanical form, but it uses the interactions of rare earth permanent magnets to penetrate certain space distances and 
materials to transfer mechanical energy. Its emergence thoroughly solved the leakage problems of some mechanical dynamic seals.

According to the structure of the magnetic force transmission, a magnetic driver can be divided into two kinds of cylinders and ring types. This driver adopted the driving form of cylinder magnetic force; its structure is shown in Figure 3. It consists of an outer rotor of the magnetic circuit (part 1), an outer permanent magnet(part 2), a tight set screw(part 3), a motor box (part 4), a motor shaft (part 5), a drive motor (part 6), o-rings (part 7), an inner rotor of the magnetic circuit (part8), an inner permanent magnet(part 9), an isolation sleeve(part 10), and a slide set (part 11). The internal magnetizer does not contact the external magnetizer directly, but realizes the seal with o-rings located at the binding surface between the isolation sleeve and the motor, which changes the dynamic seal into a static seal on the motor output shaft. Thus, it can achieve effective sealing in deepwater up to $15000 \mathrm{~m}$.

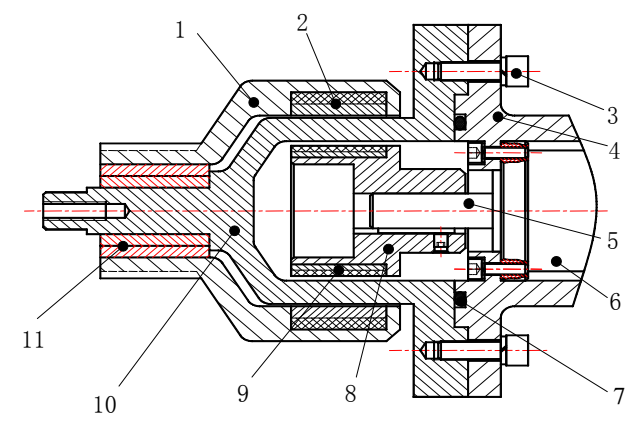

Fig. 3. Diagram of magnetic driver structure

\section{Discussion of Magnetic Coupling Principle}

The cylindrical static magnetic circuit structure is shown in Figure 4. It is composed of inside and outside magnetic rings, each of which consists of $m$ numbers of $\mathrm{N}$ and $\mathrm{S}$ poles arranged in alternate tiles in the shape of permanent magnets. Its magnetic field strength in the center of the air gap can be calculated according to the intensity superposition of the magnetic field intensity generated by two pieces of inside and outside permanent magnets. Correspondingly, it value may be written as [11]

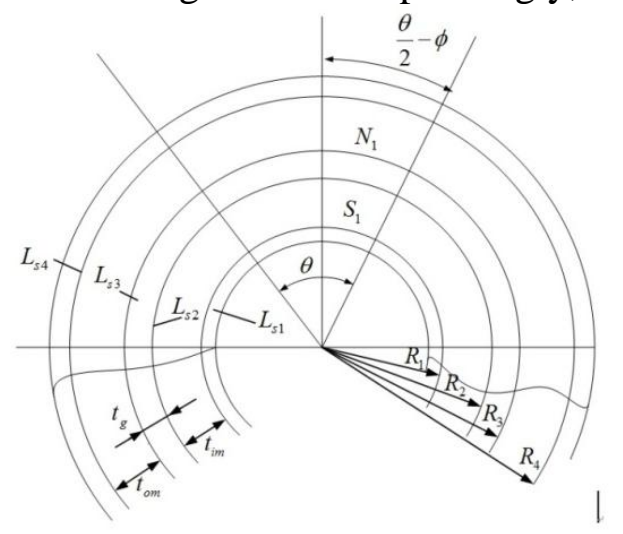

Fig. 4. Cylindrical magnetic circuit structure

$$
\begin{aligned}
& H_{i}=\frac{B_{r}}{\pi}\left[t_{g}{ }^{-1} \frac{L_{s 2} \times L_{b}}{t_{g}{\sqrt{t_{g}{ }^{2}+L_{b}{ }^{2}+L_{s 2}^{2}}}^{-}}-\right. \\
& \left.t_{g}^{-1} \frac{L_{s 1} \times L_{b}}{\left(4 t_{i m}+t_{g}\right) \sqrt{\left(4 t_{i m}+t_{g}\right)^{2}+L_{b}^{2}+L_{s 1}^{2}}}\right]
\end{aligned}
$$




$$
\begin{gathered}
H_{o}=\frac{B_{r}}{\pi}\left[t_{g}^{-1} \frac{L_{s 3} \times L_{b}}{t_{g} \sqrt{t_{g}^{2}+L_{b}{ }^{2}+L_{s 3}^{2}}}-\right. \\
\left.t_{g}^{-1} \frac{L_{s 4} \times L_{b}}{\left(4 t_{o m}+t_{g}\right) \sqrt{\left(4 t_{o m}+t_{g}\right)^{2}+L_{b}{ }^{2}+L_{s 4}^{2}}}\right] \\
H_{g}=H_{i}+H_{o}
\end{gathered}
$$

Where $H_{i}$ is the magnetic field strength generated by the inner permanent magnets; $H_{o}$ is the magnetic field strength generated by the outer permanent magnets; $H_{g}$ is the magnetic field strength working in the air gap; $B_{r}$ is the residual magnetic induction intensity of the permanent magnets; $t_{g}$ is the work air gap width; $L_{b}$ is the permanent magnet axial length; $L_{s l}$ is the inner arc length of the inner permanent magnet; $L_{s 2}$ is the outer arc length of the inner permanent magnet; $L_{s 3}$ is the inner arc length of the outer permanent magnet; $L_{s 4}$ is the outer arc length of the outer permanent magnet; $t_{i m}$ is the inner permanent magnet thickness; and $t_{o m}$ is the outer permanent magnet thickness.

The rotating process of the magnetic coupling transmission is that the inner magnetic rotor will follow it to rotate when the motor drives the outer magnetic rotor. Maximum torque can be calculated by the form:

$$
T_{\max }=\frac{1}{3} B_{r} H_{g} S_{m} R(N \cdot m)
$$

Where $S_{m}$ is the area of the permanent magnet pole interaction between the inside and outside rotors, and $R$ is the average radius of action of the inside and outside permanent magnets. For instance, when $R_{1}=1.1 \mathrm{~cm}, R_{2}=1.6 \mathrm{~cm}, R_{4}=2.65 \mathrm{~cm}, B_{r}=9300 \mathrm{Gs}, t_{g}=0.4,0.55,0.61,0.65 \mathrm{~cm}$ in Figure 4 , the data of $T_{\max }, H_{g}$ and $t_{g}$ were shown in Table 5.

Table 5. The value of Tmax and $\mathrm{Hg}$ for different $\mathrm{tg}$

\begin{tabular}{|c|c|c|}
\hline $\boldsymbol{t}_{\mathbf{g}}(\mathbf{c m})$ & $\boldsymbol{H}_{\mathbf{g}}\left(\mathbf{O}_{\mathbf{e}}\right)$ & $\boldsymbol{T}_{\mathbf{m a x}}(\mathbf{k g f} \cdot \mathbf{c m})$ \\
\hline 0.4 & 7250 & 8.37 \\
\hline 0.55 & 6079 & 14.18 \\
\hline 0.61 & 5886 & 18.78 \\
\hline 0.65 & 5754 & 13.51 \\
\hline
\end{tabular}

\section{Strength Analysis of the Isolation}

The sleeve is used to seal the inner rotor of the magnetic coupling components. The smaller the thickness of the sleeve, the less of a power loss is caused by the eddy current and the higher the transmission efficiency. The thickness of the sleeve can be written as [11]

$$
t=\frac{p D_{n}}{2\left[\sigma_{b}\right] \varphi-p}
$$

Where $P$ is shell pressure; $D_{n}$ is the inner diameter of the isolation sleeve; $\left[\sigma_{b}\right]$ is the allowable stress of the sleeve materials; and $\Phi$ is the welding coefficient, and its value takes $\Phi=1$.

For this rope drum, considering a certain intensity allowance, the external pressure is $p=18 \mathrm{MPa}$, $D_{n}=90 \mathrm{~mm}$, the material is $1 \mathrm{Gr} 18 \mathrm{Ni} 9 \mathrm{Ti}$, and its safety coefficient is $n=2$, so the available spacer thickness is $t=3.2 \mathrm{~mm}$; after rounding, the thickness is $t=4 \mathrm{~mm}$. Further analysis of the stress under the external pressure of sleeve was carried on by finite element method in Figure 5. 


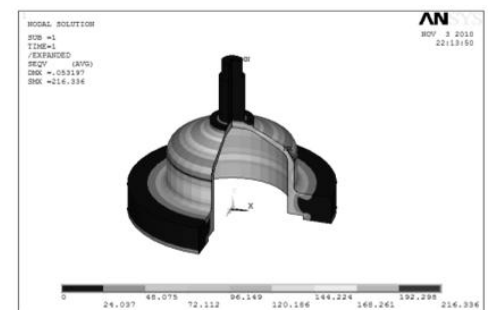

Fig. 5.The equivalent stress of the sleeve

The maximum equivalent stress located inside of the transition fillet of sleeve, which is $216 \mathrm{MPa}$ that is far less than ultimate strength of $1 \mathrm{Gr} 18 \mathrm{Ni} 9 \mathrm{Ti}, \sigma_{b}=520 \mathrm{MPa}$, so the structure of sleeve satisfies with the requirement of strength.

\section{Magnetic Driver Prototype and its Experiments}

\section{The Pressure Test of Whole Machine}

According to the requirements for experimental schemes, the test equipment includes the water tank, high-pressure water pump, and pressure test cabin, which are shown in Figure 6 (a); the magnetic driver prototype to be put into the cabin is shown in Figure $6(\mathrm{~b})$, The allow pressure could achieve to the value of $110 \mathrm{MPa}$.

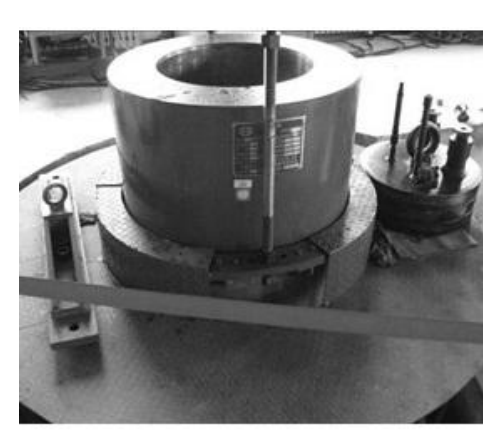

(a)

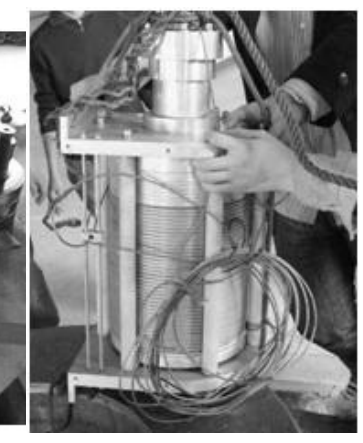

(b)

Fig. 6. The pressure test cabin and driver prototype

\section{The Test of Pulling Rope}

The driver was installed into the rope drum and the experiments were performed. The spring balance was used to simulate the tensioning force and test tensile performance of the rope drum. The results of the tensile experiment are that the ropes drum maximum tension is $338 \mathrm{~N}$, and the deflection is about $22 \mathrm{~mm}$ according to the data of the value of tension, which is less than $35 \mathrm{~mm}$ and is an allowable value of deflection under the condition of tensioning rope; therefore, the driver meets the performance requirements. But, it came out the energy loss because the spacer thickness of measured drum in Figure 1 is $t=2 \mathrm{~mm}$, which is smaller than it in the driver underwater that is $t=4 \mathrm{~mm}$. The maximum tension that is $338 \mathrm{~N}$ is lower than the measured value $376 \mathrm{~N}$ in Table 1 , the efficiency is $89.9 \%$.So, and we should optimize the structure and raise the efficiency. With the method of finite element optimization, we got the ultimate thickness was $t=3.5 \mathrm{~mm}$. Then after further tensile experiments, the maximum pulling force is $346 \mathrm{~N}$; the efficiency is $92.0 \%$, which increased by $2.1 \%$; and the deflection is about $20 \mathrm{~mm}$, which increases the safety margin.

The rope drum and its device were hung together into the sea and the rope was released while the pulled experiments were done. During the process, the divers observed underwater that the ropes drum rotated stably and pulled the rope firmly, achieving the engineering requirements. The drum before its descent into the sea is shown in Figure 7; part 1 is the rope drum, and part 2 is the measuring unit. 


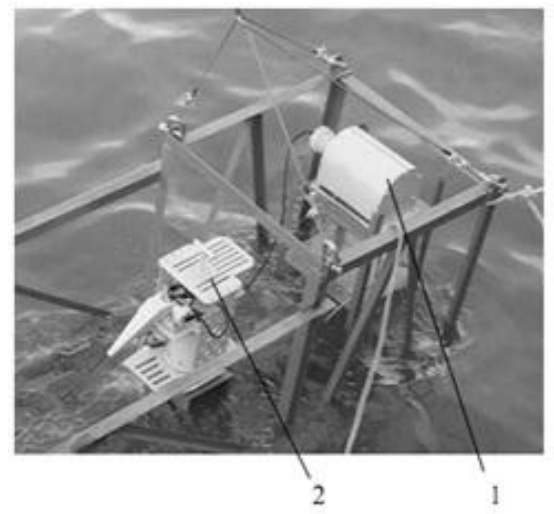

Fig. 7. Test of the rope drum in sea

\section{Conclusion}

This article carried out research on a rope drum driver made of magnetic materials; it changed the form of the dynamic seal into a static seal on the motor output shaft and solved the leakage problem of dynamic seals on motor output shafts in Deepwater. Through tests of sealing pressure and releasing the rope and then pulling it under the sea, we can conclude that the driver sealing pressure can come up to $110 \mathrm{MPa}$,equalling a1 $1000 \mathrm{~m}$ water depth; and the allow length of the rope is $30 \mathrm{~m}$. This shows that the development of the driver is feasible and reliable. At present, this device has been used in engineering construction under the sea, so it has great application value.

\section{Acknowledgements}

This material is based upon work supported by the National Science Foundation of China (Grant no. 51479043). The views expressed are authors alone.

\section{References}

[1] L.X. Shi, Z.G. Li and D.Y. Zhao, Nat. Gas. Ind, 05, 106 (2008).

[2] L.X. Zhao, X.L. Ding and Y.M. Zhang, D.I.M, 18, 132-35 (2000).

[3] Z.J. Ren, C.T, 22, 19 (2003).

[4] R.J. Wang,G.M, 2, 154 (2005).

[5] S.C. Feng, S.J. Liu, T.G. Liu et al, LUBR.ENG, 3, 1127 (2004)

[6] Z.Y. Hu, D.Z. Wu and L.Q.Wang, J. ENG.DESIGN, 12,124 (2005).

[7] H.M. Shang, OCEAN TECH, 01. 43 (2006).

[8] X.Y. Zhong, Y.G. Tan, MECH. ENG, 01, 40 (2006).

[9] M.J. JIN, Z.G. Zhang, RAIL.STANDARD DESIGN, 04, 9 (2005).

[10] W.G. Zheng, Y.K. Luo and Q. Li, Mach., 42, 53(2004).

[11] K.Z. Zhao, Beijing: Chemical Industry Press, (2009) 